\title{
Central composite design for the optimization of removal of the azo dye, Methyl Red, from waste water using Fenton reaction
}

\author{
Mahsa Azami*, Morteza Bahram and Sirous Nouri
}

Department of Chemistry, Faculty of Science, Urmia University, Urmia, Iran

\section{H R O N I C L E}

Article history:

Received October 26, 2012

Received in Revised form

March 10, 2013

Accepted 15 March 2013

Available online

15 March 2013

Keywords:

Design of experiments

Methyl red

Degradation

Fenton reaction

\begin{abstract}
A B S T R A C T
In this study the degradation of an azo dye, Methyl Red, which is used in textile industry, using Fenton reaction was studied and optimized by a chemometrics method. Fenton oxidation is one of the Advanced Oxidation Processes (AOPs), in which hydroxyl radicals are generated from Fenton's reagents $\left(\mathrm{Fe}^{2+}, \mathrm{H}_{2} \mathrm{O}_{2}\right)$. The effects of various experimental parameters in this reaction were investigated using Central Composite Design (CCD) method. The experimental design was done at five levels of operating parameters. 28 experiments, with 4 factors and 5 levels for each factor were designed. These factors (or variables) include $\left[\mathrm{Fe}^{2+}\right],\left[\mathrm{H}_{2} \mathrm{O}_{2}\right]$, [oxalate] and the reaction time. A full-quadratic polynomial equation between the percentage of dye degradation (as the response) and the studied parameters was established. After removing the nonsignificant terms from the model, response surface method was used to obtain the optimum conditions. The optimum ranges of variables were: $0.1-0.4 \mathrm{mM}$ for $\left[\mathrm{Fe}^{2+}\right], 13.5-22 \mathrm{mM}$ for $\left[\mathrm{H}_{2} \mathrm{O}_{2}\right], 1.5-2 \mathrm{mM}$ for [Oxalate], and 115-125 min for the reaction time. Also the results of extra experiments showed that these optimized values can be used for real samples and yield to high values for the response.
\end{abstract}

\section{Introduction}

\subsection{AZO DYES}

There are many branches of industries, i.e. textile, painting, leather, printing, paper production, food technology, photography etc., which use various colorants (synthetic dyes) ${ }^{1,2}$. The release of such coloured wastewaters into the environment is a dramatic source of aesthetic pollution, eutrophication and perturbations in aquatic life $^{3}$. The presence of these synthetic dyes can also make problems within the use and reuse of water streams, distribute the beautiful appearance of waters and

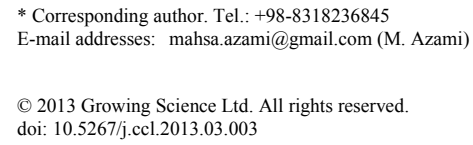


also reduce the effectiveness of microbial waste water treatment because they can be toxic to microorganisms.

Dyes in effluents absorb and scatter sunlight, which is essential for algae growth. These effluents are threat to the ecosystems due to its toxicity and resistance to destruction by biological treatment methods ${ }^{1}$. Dyes which release in wastewater should be seriously concerned because they give an undesirable color to the waters and also in some cases are themselves harmful compounds and can lead to generate harmful by-products through various reactions taking place in the waste phase ${ }^{4}$.

There is a wide variety of dyes on the market for various applications. Dyes can be classified as azo dyes, triaryl methane dyes, anthraquinone dyes, heterocyclic dyes, and phthalocyanine dyes, depending on their chemical structures. Dyes can also be characterized according to their application method as reactive dyes, direct dyes, acidic dyes, disperse dyes, and cationic dyes 5 .

Azo dyes, constituting the largest class among the synthetic colorants, are considered as the widespread environmental pollutants associated with many important industries such as textile, food colorants, printing and cosmetic manufacturing. It is estimated that approximately $800,000 \mathrm{t}$ of dyes are produced annually worldwide and about $50 \%$ of them are azo dyes ${ }^{6}$. Azo dyes are the largest class of dyes commercially used in the textile industries. Most of these compounds are highly resistant to microbial attack, and are, therefore, hardly removed from effluents by conventional biological wastewater treatments, such as activated sludge ${ }^{7}$. The release of azo dyes into the environment is of great concern, due to coloration of natural waters and toxicity, mutagenicity, and carcinogenicity of the dyes and their toxic biotransformation products ${ }^{8}$.

Methyl red, also called C.I. Acid Red 2, is an indicator dye that turns red in acidic solutions. It is an azo dye, and is a dark red crystalline powder. Methyl red is a $\mathrm{pH}$ indicator; it is red in $\mathrm{pH}$ under 4.4 , yellow in $\mathrm{pH}$ over 6.2, and orange in between, with a pKa of 5.1.

This dye is also used in textile industries and has the maximum of absorbance at $\lambda \max =432 \mathrm{~nm}$. Some of Methyl red's characteristics are shown in Table 1. Methyl Red is a harmful compound that exists in textile waste waters and has various harmful effects on human beings. For example it may cause eye or skin irritation, or inhalation may cause gastrointestinal irritation with nausea, vomiting, and diarrhea ${ }^{9,10}$.

Table 1. Some characteristics of Methyl Red.

\begin{tabular}{cccc}
\hline Name & Molecular weight $(\mathrm{g} / \mathrm{mol})$ & Chemical Formula \\
\hline Methyl Red & & 269.31 & $\mathrm{C}_{15} \mathrm{H}_{15} \mathrm{~N}_{3} \mathrm{O}_{2}$ \\
& &
\end{tabular}

\subsection{Advanced Oxidation Processes}

To avoid the dangerous accumulation of dyes in the environment, there is a need to develop effective methods for the degradation of such organic pollutants, either to less harmful compounds or, more desirable, to their complete mineralization ${ }^{11}$.

There are many different technological processes such as adsorption ${ }^{1}$, biodegradation, chemical methods (e.g. chlorination and ozonation), electrocoagulation, electrochemical reduction and oxidation, indirect Electro-oxidation with strong oxidants and photocatalytic degradation, which can be used to treat the waste waters including these synthetic dyes ${ }^{5,12}$. 
In recent years, Advanced Oxidation Processes (AOPs) have attracted attentions for the destruction of these dyes. In AOPs methods, highly reactive hydroxyl radicals, which have one unpaired electron and are very strong oxidizing agents are produced.

Fenton's oxidation is one of the AOPs methods, in which hydroxyl radicals are generated from Fenton's reagents ${ }^{1}$. Fenton reaction is already in use for industrial wastewater purification processes. It is well-known that, the most important characteristic of heterogeneous Fenton processes is the formation of $\mathrm{OH}$ radicals, which are highly oxidative, nonselective, and able to decompose many organic compounds. It is also well-accepted that the advantages of heterogeneous Fenton processes are complete mineralization of organic compounds at ambient temperature and easy separation of the heterogeneous catalysts from the treated wastewater. Because of these advantages, a lot of effort has been made in developing heterogeneous Fenton catalysts containing $\mathrm{Fe}^{2+}$ ions. The Fenton system uses ferrous ions as catalysts that react with hydrogen peroxide, producing hydroxyl radicals with powerful oxidizing abilities ${ }^{5}$.

Generated hydroxyl radicals may then react with ferrous ions, for instance, to form ferric ions, or with the organics. The oxidation products are usually low molecular weight compounds that are often more easily biodegradable or, in some instances, the organic compounds reduced to carbon dioxide and water, among other inorganics ${ }^{13}$.

The Fenton reaction has many various advantages in the degradation of pollutants, for example its reagents are environmentally safe, cheap and easy to obtain and use. The appropriate $\mathrm{pH}$ range for this reaction is $2.0-4.0$ and the best $\mathrm{pH}$ is $3^{14,15}$.

As mentioned above, the Fenton reaction is used for the treatment of azo dyes wastewaters. This reaction can be effective on the degradation of so many azo dyes. The rate of ${ }^{\circ} \mathrm{OH}$ generation and the characteristics of these dyes can influence on the percentage of decolorization ${ }^{16}$.

So it is obvious that the concentration of iron salt and $\mathrm{H}_{2} \mathrm{O}_{2}$ have an important effect on the dye removal percentage. In addition, in this study the salt of sodium oxalate is also used to catalyze the removal reaction.

\subsection{Optimization}

Optimizing refers to improving the performance of a system, a process, or a product in order to obtain the maximum benefit from it. The term optimization has been commonly used in analytical chemistry as a means of discovering conditions at which a procedure produces the best possible response $^{17}$.

In order to optimize the effective variables on decolourisation of Methyl Red, two methods can be used: one-variable-at-a-time method and experimental design techniques. In the first method it is necessary to do many experiments, spend much time and materials.

There is also a premier problem with this method. Here the interactions between the parameters are ignored and this can lead to incorrect optimized values for the variables and after doing many experiments we cannot claim that the best results are obtained. Therefore, it is obvious that a reliable and trusty method is required to gain the true optimized values. The experimental design is a method which can lead to this goal ${ }^{18-20}$.

Experimental design is used to decide which factors influence on the properties of the chemical process or desirable product. Response Surface Methodology (RSM) is a statistical method being useful for the optimization of chemical reactions and/or industrial processes and widely used for experimental design. Whenever multiple system variables may influence the outputs; RSM can be utilized to assess the relationship between dependent (response) and independent variables as well as 
to optimize the relevant processes ${ }^{21,22}$. RSM employs a low-order polynomial equation in a predetermined region of the independent variables, which are later on analyzed to locate the optimum values of independent variables to obtain the best (highest) response. Process optimization by RSM is faster for gathering experimental research results than the rather conventional, time consuming onefactor-at-a-time approach ${ }^{23,24}$.

In this research, finding optimal conditions for effective factors on degradation of Methyl Red, by Fenton reaction using an experimental design method (Central Composite Design (CCD)) was investigated.

\section{Experimental}

\subsection{Instruments}

The solutions were shaked by a MTOPO magnetic stirrer model MS3300 and the absorbance of the solutions were measured by a PG-mode T80 UV-Vis double-beam spectrophotometer (Japan) utilizing a 1-cm quartz cell. Variation of solution $\mathrm{pHs}$ was measured by a $\mathrm{pH}$-meter BEHINEH (UK).

\subsection{Reagents}

Methyl Red $\left(\mathrm{M}_{\mathrm{w}}=269.31 \mathrm{gr} / \mathrm{mol}\right), \mathrm{FeSO}_{4} \cdot 7 \mathrm{H}_{2} \mathrm{O}\left(\mathrm{M}_{\mathrm{w}}=278 \mathrm{gr} / \mathrm{mol}\right), \mathrm{H}_{2} \mathrm{O}_{2}(\mathrm{a}=30 \% \mathrm{v} / \mathrm{v}, \mathrm{d}=1.11$ $\left.\mathrm{kg} / \mathrm{m}^{3}\right)$ and $\mathrm{Na}_{2} \mathrm{C}_{2} \mathrm{O}_{4}\left(\mathrm{M}_{\mathrm{w}}=134 \mathrm{gr} / \mathrm{mol}\right)$, were used in analytical grade (Merck) and all of the solution were prepared with fresh distilled water.

The solution of $\mathrm{Fe}^{2+}$ ion was prepared several times during a day and was used immediately to prevent the exchange of $\mathrm{Fe}^{2+}$ ion to $\mathrm{Fe}^{3+}$ ion.

To adjust the final $\mathrm{pH}$ of the solutions, the solutions of $\mathrm{H}_{2} \mathrm{SO}_{4}(0.01 \mathrm{M})$ and $\mathrm{NaOH}(0.01 \mathrm{M})$ were prepared and used.

\subsection{Statistical Software}

Essential Regression and Experimental Design for Chemists and Engineers (EREGRESS), as MS Excel Add-In software, was used to design the experiments and to model and analyze the results.

\subsection{Design of Experiments (DOE)}

By using CCD method, 28 experiments (including 4 repetitions at central point) were designed. The factors (variables) were: $\left[\mathrm{Fe}^{2+}\right],\left[\mathrm{H}_{2} \mathrm{O}_{2}\right]$, [oxalate] and the reaction time. Although the initial concentration of Methyl Red might be important, but as mentioned, this factor was spotted at the constant value of $0.015 \mathrm{mM}$. This value for the dye concentration was selected from calibration curve of Methyl Red (the middle point of the linear range in spectrophotometeric determination). For each factor, 5 levels were defined. These values are coincided on the codes: $-2,-1,0,+1$ and +2 and are shown in Table 2.

Table 2. The variables and values used for CCD

\begin{tabular}{ccccccc}
\hline Variable & Name & Low $(-2)$ & -1 & 0 & +1 & High $(+2)$ \\
\hline $\mathrm{F} 1$ & {$\left[\mathrm{Fe}^{2+}\right] / \mathrm{mM}$} & 0 & 0.25 & 0.5 & 0.75 & 1 \\
$\mathrm{~F} 2$ & {$\left[\mathrm{H}_{2} \mathrm{O}_{2}\right] / \mathrm{mM}$} & 0 & 5.5 & 11 & 16.5 & 22 \\
$\mathrm{~F} 3$ & {$[\mathrm{Oxa} a \mathrm{ate}] / \mathrm{mM}$} & 0 & 0.5 & 1 & 1.5 & 2 \\
$\mathrm{~F} 4$ & Time $/ \mathrm{min}$ & 5 & 35 & 65 & 95 & 125 \\
\hline
\end{tabular}




\subsection{Preparing the Solutions}

At first, it is necessary to find the linear range of Methyl Red as its calibration curve. The calibration curve represents the absorbance values versus concentration values. A spectrum of a solution of Methyl Red which is taken in visible area, shows the maximum wavelength of this dye at $432 \mathrm{~nm}$ (Figure 1).Then, several samples with definite concentrations were made and their absorbencies were measured at $432 \mathrm{~nm}$ and the linear range of Methyl Red in spectrophotometric detection was specified (Figure 2). A concentration in middle points of this curve was selected and used for the preparing the dye solution in all experiments $(0.015 \mathrm{mM})$.

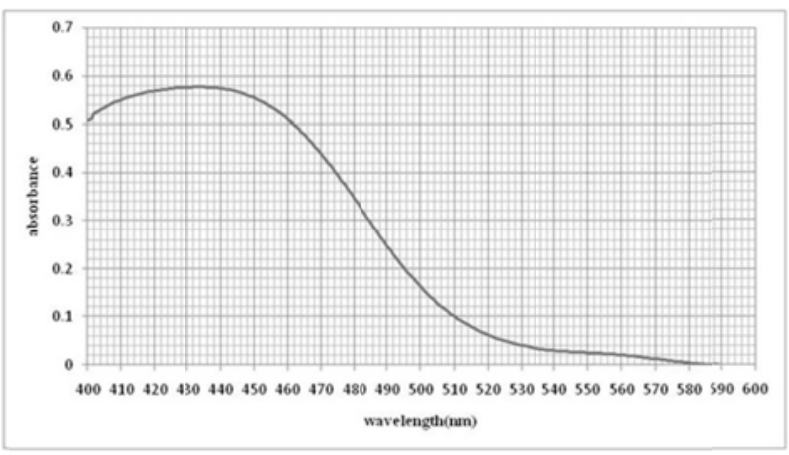

Fig 1. Maximum absorbance of Methyl Red

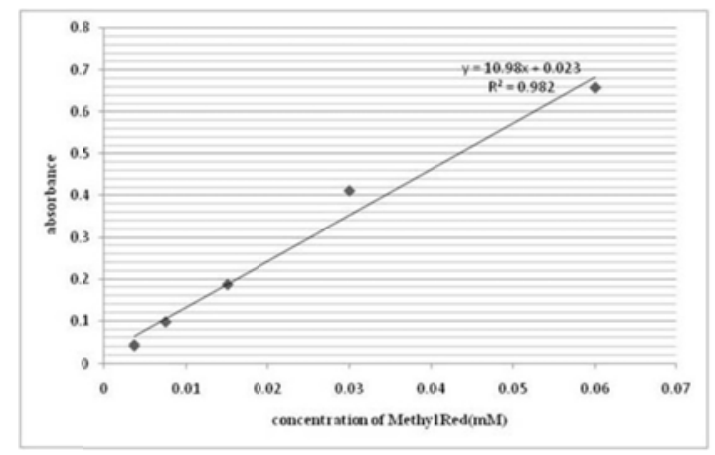

Fig 2. The calibration curve of Methyl Red (432 nm)

According to Table 3, in each experiment, two solutions are needed; A blank solution and a sample solution. The concentration of Methyl Red, $\mathrm{H}_{2} \mathrm{O}_{2}, \mathrm{Fe}^{2+}$ and oxalate are extracted from the Table of DOE (Table 2 and Table 3).

The $\mathrm{pH}$ of solution was adjusted in 3.5 , using $\mathrm{H}_{2} \mathrm{SO}_{4}(0.01 \mathrm{M})$ and $\mathrm{NaOH}(0.01 \mathrm{M})$, because the appropriate $\mathrm{pH}$ for the Fenton reaction is in the range of 3-5. For each reaction, also a blank sample was prepared which was including the same concentration of Methyl Red and $\mathrm{Fe}^{2+}$, but without $\mathrm{H}_{2} \mathrm{O}_{2}$ and oxalate ions. The aim of preparing blank solution for each experiment is to consider the effect of passing the time on spontaneously probable degradation of Methyl Red. Also the aim of adding the $\mathrm{Fe}^{2+}$ in blank solution is to neutralize the eventual effect of $\mathrm{Fe}^{2+}$ s $\mathrm{s}$ absorption on the absorption of main solution. These samples were shacked with a magnetic stirrer during reaction.

After the prescribed needful time for each reaction (Table 3), the absorbance of these two solutions were measured with the Uv-Vis spectrophotometer.

\section{Results and Discussion}

\subsection{Data Analysis}

After doing the experiments, the percentage of dye degradation for each experiment was calculated from Eq. (1), which was spotted as the response.

$$
\% \text { degradation }=\left[1-\left(\frac{\text { sample absorbance }}{\text { blank absorbance }}\right)\right] * 100
$$

Table 3 shows the percentage of dye degradation for each experiment. Among these 28 experiments, 4 experiments were repetition of central point (numbers 5, 15, 20, 28). These are the experiments in which, all of the factors are in the centric point of their values. Closeness of the responses of these 4 experiments can be a sign of the accuracy of the experiment process. The relation between the collected response and the variables conforms from the following polynomial equation - a full quadratic model- (Eq. (2)): 
Response $=\mathrm{b}_{0}+\mathrm{b}_{1} * \mathrm{~F}_{1}+\mathrm{b}_{2} * \mathrm{~F}_{2}+\mathrm{b}_{3} * \mathrm{~F}_{3}+\mathrm{b}_{4} * \mathrm{~F}_{4}+\mathrm{b}_{5} * \mathrm{~F}_{1} * \mathrm{~F}_{1}+\mathrm{b}_{6} * \mathrm{~F}_{2} * \mathrm{~F}_{2}+\mathrm{b}_{7} * \mathrm{~F}_{3} * \mathrm{~F}_{3}$

$+\mathrm{b}_{8} * \mathrm{~F}_{4} * \mathrm{~F}_{4}+\mathrm{b}_{9} * \mathrm{~F}_{1} * \mathrm{~F}_{2}+\mathrm{b}_{10} * \mathrm{~F}_{1} * \mathrm{~F}_{3}+\mathrm{b}_{11} * \mathrm{~F}_{1} * \mathrm{~F}_{4}+\mathrm{b}_{12} * \mathrm{~F}_{2} * \mathrm{~F}_{3}+\mathrm{b}_{13} * \mathrm{~F}_{2} * \mathrm{~F}_{4}+\mathrm{b}_{14} * \mathrm{~F}_{3} * \mathrm{~F}_{4}$

In this equation $F 1, F 2, F 3$ and F4 show the variables and the $b_{0}-b_{14}$ are the coefficient values obtained by multiple linear regression (MLR).

Table 3. The coded values designed by $\mathrm{CCD}$ and responses

\begin{tabular}{cccccc}
\hline Run & {$[\mathrm{Fe} 2+]$} & {$\left[\mathrm{H}_{2} \mathrm{O}_{2}\right]$} & {$[$ Oxalate $]$} & Time & \% Degradation \\
\hline 1 & -1 & -1 & 1 & -1 & 70.10 \\
2 & 1 & 1 & 1 & 1 & 60.53 \\
3 & -1 & 1 & 1 & -1 & 83.09 \\
4 & 1 & 1 & -1 & -1 & 49.03 \\
5 & 0 & 0 & 0 & 0 & 60.00 \\
6 & -1 & -1 & 1 & 1 & 70.75 \\
7 & 1 & 1 & 1 & -1 & 71.20 \\
8 & 0 & 0 & -2 & 0 & 39.24 \\
9 & 1 & -1 & -1 & -1 & 39.57 \\
10 & 0 & 0 & 0 & 2 & 74.48 \\
11 & -1 & 1 & -1 & -1 & 63.08 \\
12 & 0 & 2 & 0 & 0 & 69.33 \\
13 & -1 & 1 & -1 & 1 & 79.41 \\
14 & -1 & -1 & -1 & 1 & 70.19 \\
15 & 0 & 0 & 0 & 0 & 61.25 \\
16 & 0 & 0 & 2 & 0 & 69.57 \\
17 & 0 & 0 & 0 & -2 & 78.42 \\
18 & 1 & -1 & -1 & 1 & 35.93 \\
19 & -2 & 0 & 0 & 0 & 52.15 \\
20 & 0 & 0 & 0 & 0 & 61.29 \\
21 & 0 & -2 & 0 & 0 & 50.14 \\
22 & -1 & 1 & 1 & 1 & 77.33 \\
23 & 1 & -1 & 1 & -1 & 35.16 \\
24 & -1 & -1 & -1 & -1 & 74.38 \\
25 & 2 & 0 & 0 & 0 & 14.65 \\
26 & 1 & 1 & -1 & 1 & 72.50 \\
27 & 1 & 0 & 1 & 1 & 57.41 \\
28 & & & 0 & 0 & 66.67 \\
\hline & & 0 & & &
\end{tabular}

The analysis of variance (ANOVA) and least squares techniques were used to evaluate the statistical significance of the constructed models. The ANOVA consists of determining which factor(s) significantly affect the response, using a Fisher's statistical test (F-test). The significance and the magnitude of the estimated coefficients of each variable and all their possible interactions on the response variable(s) are determined. Effects with a confidence level less than $95 \%$ (effects with a p-value higher than 0.05) were discarded and pooled into the error term and a new analysis of variance was performed for the reduced model. Note that the p-value represents a decreasing index of the reliability of a result. Four replicates of the central points were performed to estimate the experimental error. In order to show the fitness of the model, regression coefficient $(\mathrm{R})$ maybe be used. However, the adjusted regression coefficient $\left(\mathrm{R}_{\mathrm{adj}}\right)$ and the prediction regression coefficient $\left(R_{\text {pred }}\right)$ is better criteria than the absolute regression coefficient $(R)$. Since the regression coefficient $(\mathrm{R})$ always decreases when a regression variable is eliminated from the model, in statistical modelling the $R_{a d j}$, which takes the number of regression variables into account, is usually selected. 
In addition, $\mathrm{R}_{\text {pred, }}$ which indicates the predictive power of the model, is chosen for the same reason. This parameter was approximated using prediction error sum of squares (PRESS) that is calculated from residuals. So, the regression coefficient $(R)$, adjusted $R\left(R_{a d j}\right)$, and $R$ for prediction $\left(R_{\text {pred }}\right)$ together are very convenient to get a quick view of the overall fit and the prediction power of a constructed model ${ }^{25}$.

In order to find the important factors and build a model to optimize the procedure, we started with a full quadratic model including all terms in (Eq. (2). Then by back elimination process, those terms, which were not significant enough were eliminated from the equation. These terms included the variables or the interactions, which had no effect or very low effect on the response. Each term which its $\mathrm{P}$-value is more than 0.05 , is removed from the main equation. The final equation between the response and the variables is shown in Eq. (3). As it is obvious, the concentration of $\mathrm{Fe}^{2+}$ ion, and the interactions between the time of Fenton reaction and $\mathrm{Fe}^{2+}$ ion concentration, oxalate ion concentration and $\mathrm{H}_{2} \mathrm{O}_{2}$ concentration are significant and have important effects on the percentage of dye removal. Importance of the interactions is shown graphically in Figure 3.

$\%$ Degradation $=\mathrm{b} 0+\mathrm{b} 1 *[$ Oxalate $]+\mathrm{b} 2 *$ Time $+\mathrm{b} 3 *\left[\mathrm{Fe}^{2+}\right] *\left[\mathrm{Fe}^{2+}\right]+\mathrm{b} 4 *\left[\mathrm{Fe}^{2+}\right] *\left[\mathrm{H}_{2} \mathrm{O}_{2}\right]$

+ b5*Time*Time

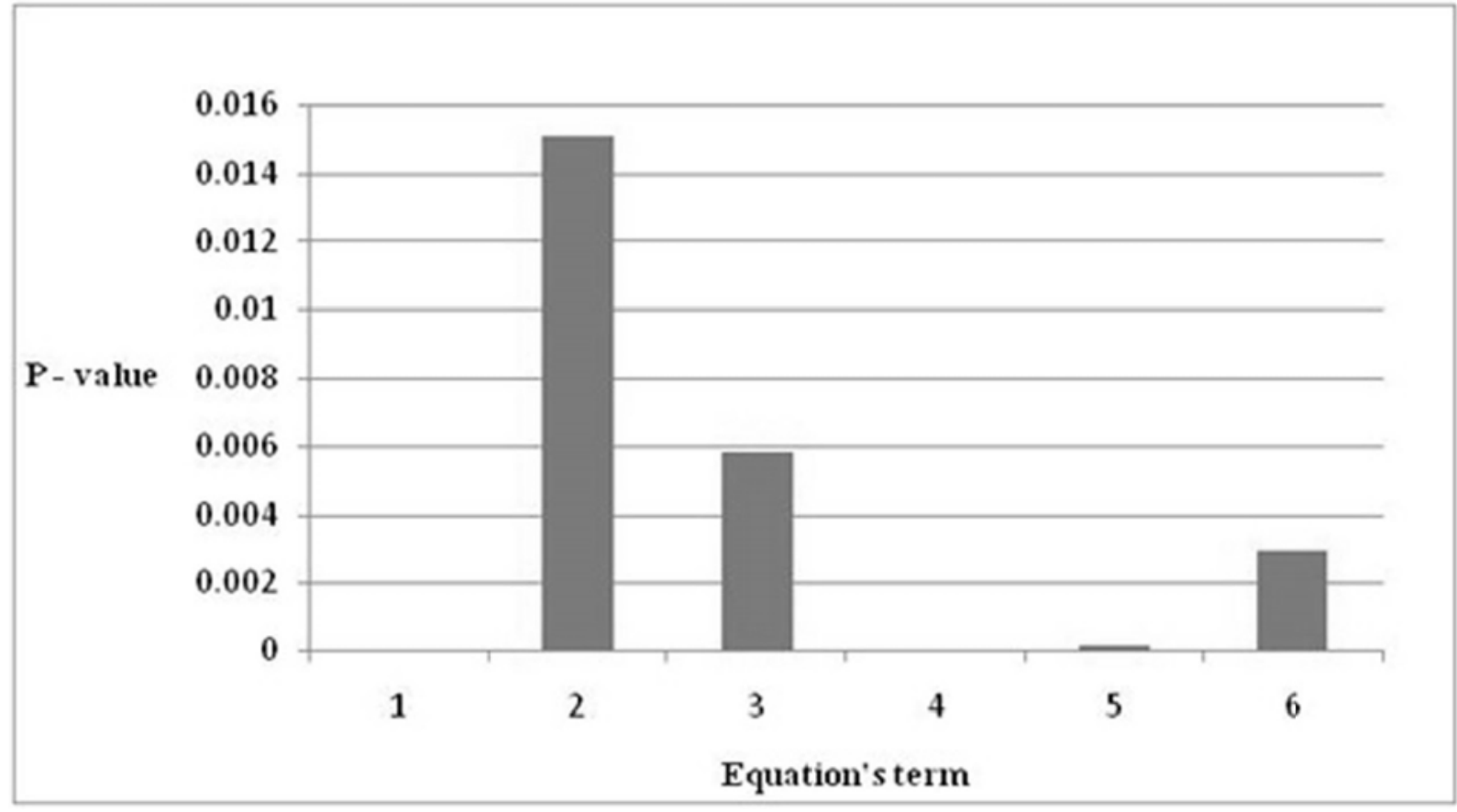

Fig. 3. P-values for the terms in reduced polynomial equation

The reduced model using significant linear, quadratic and interaction parameters is defined in the form of above polynomial Eq. (3).

There were not large differences between $\mathrm{R}$ values which revealed that the experimental data shows a good fit to the quadratic equations and therefore an acceptable model has been achieved.

\subsection{Response surface and selection of optimized values}

After the analysis of data, response surfaces of full quadratic model between the response and the variables were depicted. Via these surfaces the relations between the percentage of dye degradation and effective factors are graphically given. These surfaces are shown in Figure 4. The optimized 
ranges for each factor that leads to the best response (the highest percentage of dye degradation), were extracted from these surfaces. The optimized ranges are presented in Table 4.

Table 4. The optimized ranges for 4 variables

\begin{tabular}{cc}
\hline Variable & Optimized range \\
\hline$\left[\mathrm{Fe}^{2+}\right] / \mathrm{Mm}$ & $0.1-0.4$ \\
{$\left[\mathrm{H}_{2} \mathrm{O}_{2}\right] / \mathrm{mM}$} & $13.5-23$ \\
{$[$ Oxalate $] / \mathrm{mM}$} & $1.5-2$ \\
{$[$ time $] / \mathrm{min}$} & $115-125$ \\
\hline
\end{tabular}

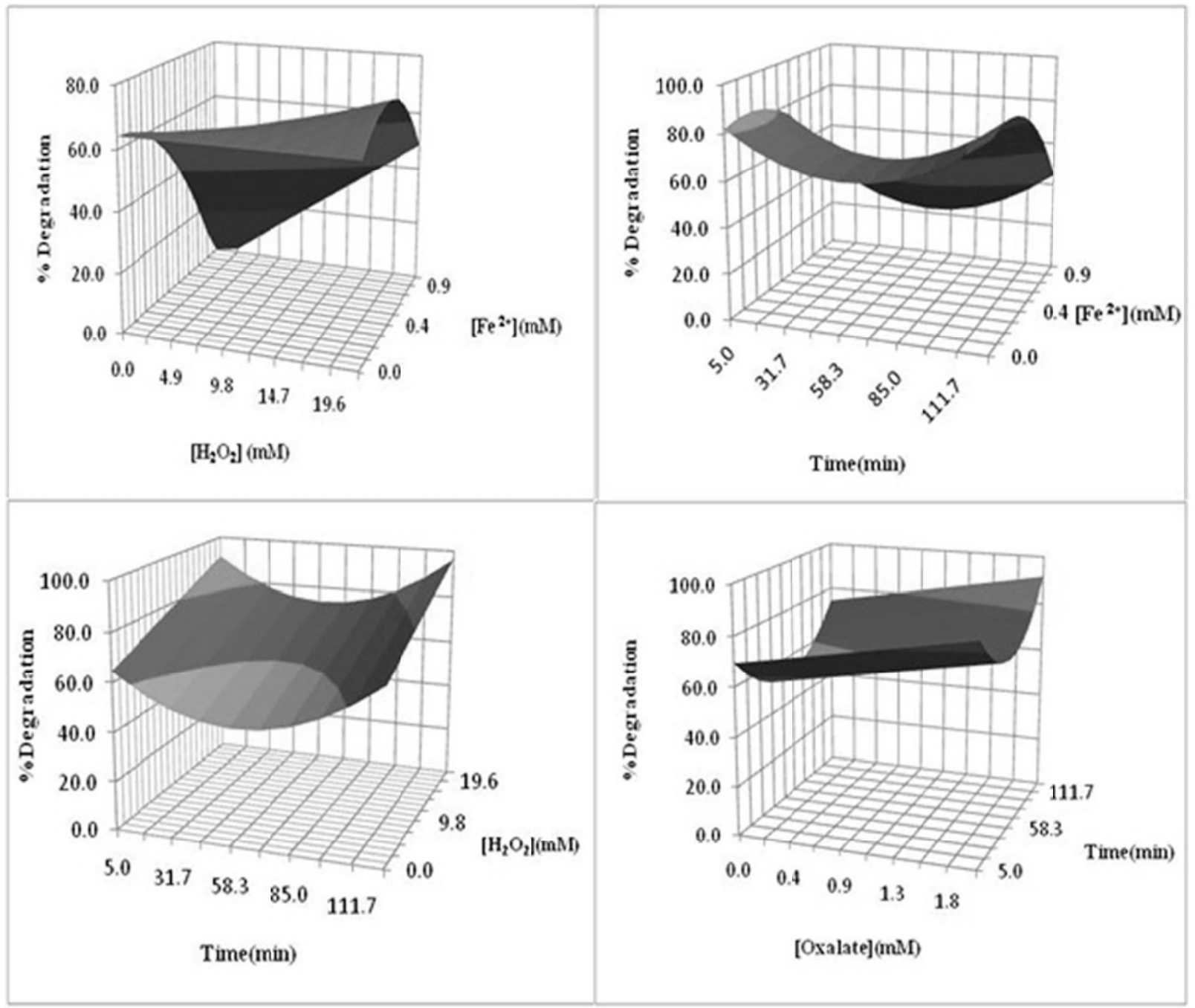

Fig. 4. Response surfaces of full quadratic model between percentage of dye degradation and four variables and the interaction between variables

In order to test the accuracy of obtained optimized ranges of variables, it is necessary to select the middle point of each optimum range and repeat the experiments at these values. Five repetitive experiments were done in these conditions. As we can see, all the responses were much closed to each other. These results can satisfy that the obtained optimized ranges for this reaction, can lead to high percentages of dye degradation (Table 5). 
Table 5. The results of 5 repetitions of optimum conditions

\begin{tabular}{cccccccc}
\hline Run & $\begin{array}{c}{\left[\mathbf{F e}^{2+}\right]} \\
\text { /mM }\end{array}$ & $\begin{array}{c}{\left[\mathbf{H}_{\mathbf{2}} \mathbf{O}_{2}\right] /} \\
\mathbf{m M}\end{array}$ & $\begin{array}{c}{[\text { Oxalate]// }} \\
\mathbf{m M}\end{array}$ & $\begin{array}{c}\text { Time/ } \\
\text { min }\end{array}$ & $\begin{array}{c}\text { Blank } \\
\text { Absorbance }\end{array}$ & $\begin{array}{c}\text { Sample } \\
\text { Absorbance }\end{array}$ & $\begin{array}{c}\text { \% } \\
\text { Degradation }\end{array}$ \\
\hline $\mathbf{1}$ & 0.25 & 17.75 & 1.75 & 120 & 0.109 & 0.002 & 81.65 \\
$\mathbf{2}$ & 0.25 & 17.75 & 1.75 & 120 & 0.113 & 0.023 & 79.64 \\
$\mathbf{3}$ & 0.25 & 17.75 & 1.75 & 120 & 0.115 & 0.021 & 81.73 \\
$\mathbf{4}$ & 0.25 & 17.75 & 1.75 & 120 & 0.110 & 0.019 & 82.72 \\
$\mathbf{5}$ & 0.25 & 17.75 & 1.75 & 120 & 0.112 & 0.02 & 82.14 \\
\hline
\end{tabular}

The relative standard deviation (RSD) of these repeated experiments was estimated for the responses to study the precision of the experiments and closeness of the responses (Eq. (4) and Eq. (5)).

$s=\sqrt{\frac{\left(x_{i}-\bar{x}\right)^{2}}{n-1}}=1.178$

$\mathrm{RSD} \%=\frac{\mathrm{s}}{\overline{\mathrm{x}}} * 100=1.44 \%$

\subsection{Effect of Variables}

\subsubsection{Effect of $\left[\mathrm{Fe}^{2+}\right]$}

With increasing the $\left[\mathrm{Fe}^{2+}\right]$, an increasing pattern is observed in the percentage of dye degradation; but increasing of the $\left[\mathrm{Fe}^{2+}\right]$ more than a specific limit, can lead to fix or decrease the percentage of dye degradation. Because with the increasing of $\left[\mathrm{Fe}^{2+}\right]$, an increasing pattern in the ${ }^{\circ} \mathrm{OH}$ generation takes place; but the generation of ${ }^{\circ} \mathrm{OH}$ in high values, can cause the undesirable reactions between 'OH and present $\mathrm{H}_{2} \mathrm{O}_{2}$ in the solution.

\subsubsection{Effect of $\left[\mathrm{H}_{2} \mathrm{O}_{2}\right]$}

According to Eq.(C.2) and Figure 4 it is obvious that there is an interaction between the two factors $\left[\mathrm{H}_{2} \mathrm{O}_{2}\right]$ and $\left[\mathrm{Fe}^{2+}\right]$. In low concentrations of $\left[\mathrm{Fe}^{2+}\right]$, the $\left[\mathrm{H}_{2} \mathrm{O}_{2}\right]$ has no significant effect on the percentage of dye degradation; but with increasing the concentrations of $\left[\mathrm{Fe}^{2+}\right]$, the effect of $\left[\mathrm{H}_{2} \mathrm{O}_{2}\right]$ on the percentage of dye degradation increases (in the form of a linear function).

\subsubsection{Effect of [oxalate]}

According to Eq.(C.2) and Figure 4 there is no interaction between the concentrations of oxalate and other factors. Increasing the concentration of sodium oxalate (as a catalyst), in the form of a linear function, can lead to increasing the percentage of dye degradation.

\subsubsection{Effect of time}

According to Figure 4 two ranges are gained as optimized values for the factor of time (5-10 min and $115-125 \mathrm{~min})$. But it is clear that the second range (115-125 $\mathrm{min})$ has led to higher responses than the other range (5-10 $\mathrm{min})$. Therefore the second range is selected as the best value of time in this reaction.

\subsubsection{Interaction parameters}

The Eq. (3) and the response surfaces (Figure 4) show that the only important existent interaction between the four effective above factors, is the interaction between $\left[\mathrm{Fe}^{2+}\right]$ and $\left[\mathrm{H}_{2} \mathrm{O}_{2}\right]$. Other interactions were non-significant or low-significant which are discarded from the main equation. 


\subsection{Real Sample}

After gaining the optimized values for the effective variables and testing them with extra experiments, these experimental qualifications were applied on a real sample which was derivative from the waste water of a local textile factory (Urmia, Iran).

The results showed that the optimum values have been achieved by $\mathrm{CCD}$ and response surface method and that could lead to acceptable responses. Figure 5 represents the spectrum of real sample before and after treatment by the proposed method. The degradation percentage $76.45 \%$ ( using Eq. (B)) was achieved for removal of Methyl Red in real sample.

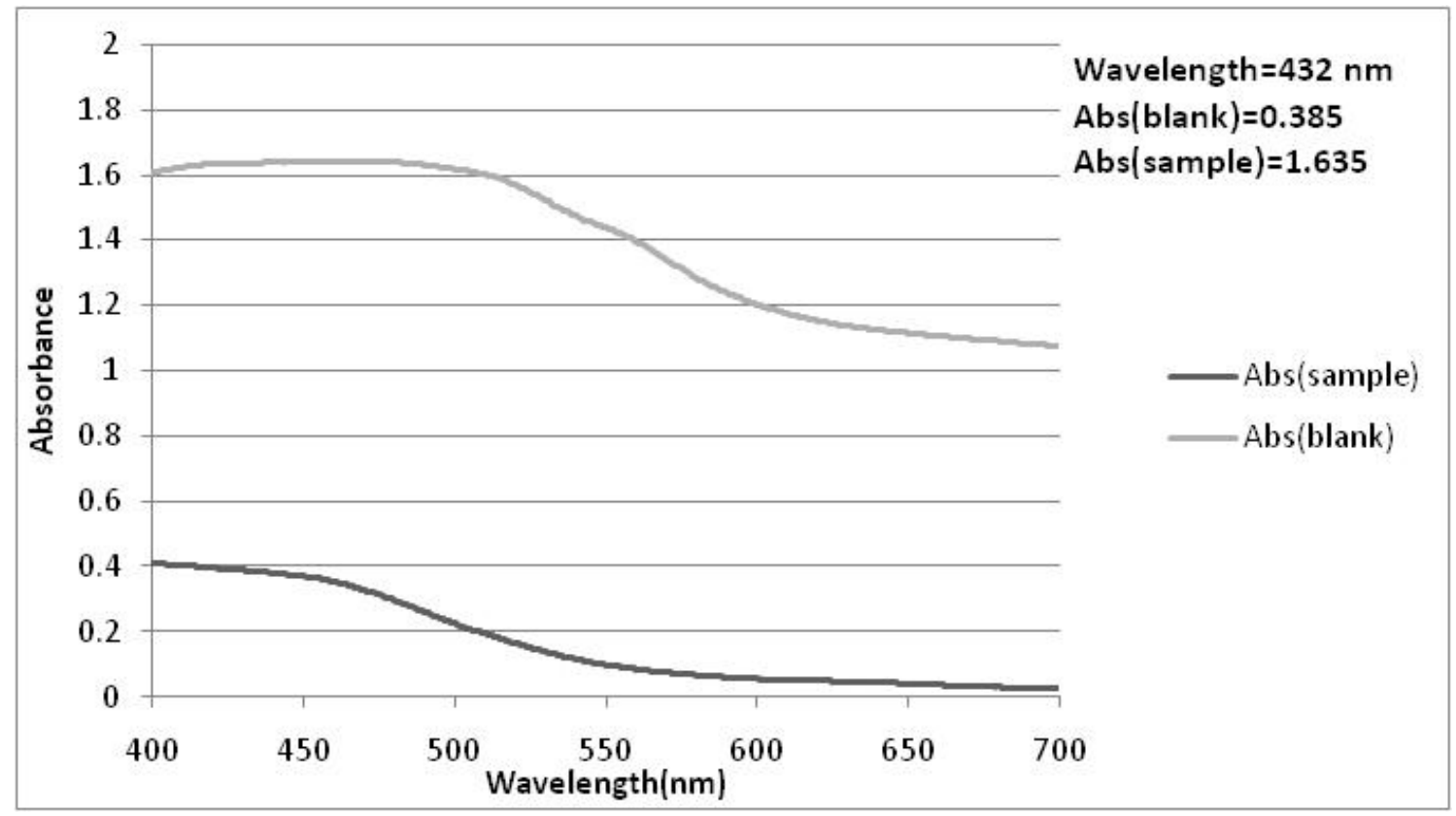

Fig. 5. Comparison between the spectrum of real sample before using Fenton reaction (blank) and after using Fenton reaction(sample)

\section{Conclusion}

In order to obtain the optimized conditions for removal of Methyl Red from a textile wastewater, 28 experiments (including 4 repetitions of central points) using CCD method were designed. The optimized values were: $0.1-0.4 \mathrm{mM}$ for $\left[\mathrm{Fe}^{2+}\right], 13.5-22 \mathrm{mM}$ for $\left[\mathrm{H}_{2} \mathrm{O}_{2}\right], 1.5-2 \mathrm{mM}$ for [Oxalate], and 115-125 min for the reaction time. These conditions were applied on a real sample and an acceptable response was achieved ( $76.45 \%$ for dye removal).

\section{References}

1. Gomathi Devi L., Girish Kumar S., Mohan Reddy K., Munikrishnappa C. (2009) Photo degradation of Methyl Orange an azo dye by Advanced Fenton Process using zero valent metallic iron: Influence of various reaction parameters and its degradation mechanism. J. Hazard. Mater.,164, 459-467.

2. Hameed B.H., Lee T.W. (2009) Degradation of malachite green in aqueous solution by Fenton process. J. Hazard. Mater., 164, 468-72.

3. Garc'1a-Monta no J., P'erez-Estrada L., Oller I., Maldonado M. I., Torrades F., Peral J. (2008) Pilot plant scale reactive dyes degradation by solar photo-Fenton and biological processes. $J$. Photochem. Photobiol., 195, 205-214. 
4. Khataee A. R., Zarei M., Moradkhannejhad L. (2010) Application of response surface methodology for optimization of azo dye removal by oxalate catalyzed photoelectro-Fenton process using carbon nanotube-PTFE cathode. Desalination., 258,112-119.

5. Rodriguez A., Ovejero G., Sotelo J. L., Mestanza M., Garcia J. (2010) Heterogeneous Fenton catalyst supports screening for mono azo dye degradation in contaminated wastewaters. Ind. Eng. Chem. Res., 49, 498-505.

6. Zhou M., He J. (2007) Degradation of azo dye by three clean advanced oxidation processes: Wet oxidation, electrochemical oxidation and wet electrochemical oxidation-A comparative study. Electrochim. Acta., 53,1902-1910.

7. Pourbabaee A., Malekzadeh F., Sarbolouki M. N., Mohajeri A. (2005) Decolorization of Methyl Orange (as a model azo dye) by the newly discovered Bacillus Sp. Iran. J. Chem. Chem. Eng., 24, 41-45.

8. Jain R., Varshney S., Sikarwar S. (2007) Electrochemical techniques for the removal of Reactofix Golden Yellow 3 RFN from industrial wastes J. Iran. Chem. Soc.., 313, 248-253.

9. Wang H., Niu J., Long X., He Y. (2008) Sonophotocatalytic degradation of methyl orange by nano-sized $\mathrm{Ag} / \mathrm{TiO} 2$ particles in aqueous solutions. Ultrason. Sonochem., 15, 387-392.

10.Diagne M., Oturan N., Oturan M. A. (2007) Removal of methyl parathion from water by electrochemically generated Fenton's reagent. Chemosphere, 66, 841-848.

11.Zarei M., Niaei A., Salari D., Khataee A. R. (2010) Removal of four dyes from aqueous medium by the peroxi-coagulation method using carbon nanotube-PTFE cathode and neural network modelling, J. Electroanal. Chem., 639, 167-174.

12. Daneshvar N., Hejazi M.J., Khataee A.R., Rangarangy B. (2004) Photocatalytic degradation of an organophosphorus pesticide phosalone in aqueous suspensions of titanium dioxide. J. Environ. Heal. B., 39, 285-296.

13.Kurbus T., March Slokar Y., Majcen Le Marechal A., Voncinab D. B. (2003) The use of experimental design for the evaluation of the influence of variables on the $\mathrm{H}_{2} \mathrm{O}_{2} / \mathrm{UV}$ treatment of model textile waste water. Dyes and Pigments, 58, 171-178.

14.Sun S. P., Li C. J., Sun J. H., Shi S. H., Fan M. H., Zhou Q. (2009) Decolorization of an azo dye Orange $\mathrm{G}$ in aqueous solution by Fenton oxidation process: Effect of system parameters and kinetic study. J. Hazard. Mater., 161,1052-1057.

15.Gutowska A., Kaluzna-Czaplinska J., Jozwiak W.K. (2007) Degradation mechanism of Reactive Orange 113 dye by $\mathrm{H}_{2} \mathrm{O}_{2} / \mathrm{Fe} 2 \mathrm{p}$ and ozone in aqueous solution. Dyes and Pigments, 74, 41-46.

16.Kurbusa T., Slokara Y. M., Majcen Le Marechal A., Voncinab D. B. (2003)The use of experimental design for the evaluation of the influence of variables on the $\mathrm{H}_{2} \mathrm{O}_{2} / \mathrm{UV}$ treatment of model textile waste water. Dyes and Pigments, 58, 171-178.

17.Arau' jo P.W., Brereton R.G. (1996) Experimental design II. Optimization. Trends Anal. Chem., $15,63-70$.

18.Brandvik P. J. (1998) Statistical simulation as an effective tool to evaluate and illustrate the advantage of experimental designs and response surface methods. Chemomet. Intell. Lab. Syst., 42, 51-61.

19.Parida K.M., Sahu N., Biswal N.R., Naik B., Pradhan A.C. (2008) Preparation, characterization, and photocatalytic activity of sulfate-modified titania for degradation of methyl orange under visible light. J. Iran. Chem. Soc., 318, 231-237.

20.Lau W. J., and Ismail A. F. (2010) Application of response surface methodology in pes/speek blend NF membrane for dyeing solution treatment. Membrane Water Treatment, 1, 49-60.

21.Daneshvar N., Salari D., Khataee A.R. (2003) Photocatalytic degradation of azo dye acid red 14 in water: investigation of the effect of operational parameters, J. Photochem.Photobiol.,157,111116.

22.Kincl M., Turk S., Vrecer F. (2005) Application of experimental design methodology in development and optimization of drug release method, Int. J. Pharm., 291, 39-49. 
23.Zhang X., Wang R., Yang X., and Yu J. (2007) Central composite experimental design applied to the catalytic aromatization of isopherone to 3,5-xylenol. Chemometr. Intell. Lab. Sys., 89, 45-50.

24.Avila A., Sanchez E.I., Gutierrez M.I. (2005) Optimal experimental design applied to the dehydrochlorination of poly(vinyl chloride), Chemom. Intell. Lab. Syst. 77, 247-250.

25.Gorji S., Bahram M. (2010) Experimental design for the study and optimization of the effect of different surfactants on the spectrophotometric determination of sulfide based on phenothiazine dye production. Anal. Methods, 2, 948-953. 